\title{
Predicting Autonomic Dysfunction in Anxiety Disorder from ECG and Respiratory Signals Using Machine Learning Models
}

\author{
https://doi.org/10.3991/ijoe.v17i07.22581
}

\author{
Abhilash Saj George, Arjun Vijayanatha Kurup, Parthasarathy Balachandran, \\ Manjusha Nair, Siby Gopinath, Anand Kumar, Harilal Parasuram ( $\left.{ }^{\varpi}\right)$ \\ Amrita Institute of Medical Sciences, Kerala, India \\ Amrita Vishwa Vidyapeetham, Amritapuri, India \\ harilalpeaims.amrita.edu
}

\begin{abstract}
Anxiety is a cognitive, behavioural, and biological response that prepares the individual to handle the stresses and conflicts of everyday life. The excessive appearance of this biological response is diagnosed as an anxiety disorder, which is often associated with Autonomic dysfunction (ADy). ADy is difficult to study in clinics with very few parameters available. Detection of ADy may not be possible/difficult in anxiety disorder with the existing method. In this study, we built machine learning models to identify ADy in subjects with anxiety using properties extracted from ECG and respiratory signals. For each dataset, statistical and frequency domain features were estimated from ECG and respiratory signals. Supervised machine learning (ML) algorithms were used to classify the subjects. Out of 23 features estimated, 11 were found to be statistically significant for the classification. We segmented the signals into 5, 10, and 30 minutes intervals to build generalized models. To overcome data imbalance, ensemble techniques like boosting was used. The highest accuracy was obtained in the SVM, Random Forest and Gradient Boosting classifiers (cross-validation accuracy of $82.2 \%, 81.64 \%$ and $79.06 \%$ and; AUC of $0.81,0.76$ and 0.84 ) for 10 and 30 minutes segmented datasets. Our results showed that the features extracted from the ECG signal are a good marker for diagnosing ADy in patients with anxiety disorder. Further, a deep neural network-based model can be implemented that may achieve better accuracy for classification provided with the cost of a large number of datasets and computation time.
\end{abstract}

Keywords-Autonomic dysfunctions, Anxiety disorder, Heart rate variability, Respiratory rate variability, Support Vector, Machine Learning

\section{Introduction}

Autonomic Dysfunction (ADy) is a largely neglected diagnostic part in neurology unlike the central nervous system and peripheral nervous system. Identification of ADy is very essential for the early detection of mental disorders like anxiety and serious events like coronary heart disease [1]. ADy is difficult to study in clinics with very few 
parameters available, unlike diseases of the central nervous system and peripheral nervous system. In this study, we are attempting to analyze the autonomic nervous system through parameters including heart rate variability and respiratory rate variability.

Anxiety is a normal biological response of our body that prepares an individual to handle the stresses and conflicts of daily life. This response also enhances the detection of threats in situations or environments that are potentially dangerous [2]. For the assessment of such extreme conditions (anxiety disorder), screening questions and batteries were developed and often used in clinics [3]. The Hamilton Anxiety Rating Scale (HAM-A) was one of the first rating scales produced to measure the severity of anxiety symptoms, and it is still commonly used in clinical and research settings [3]. The scale assesses both psychic and somatic anxiety (mental agitation and mental distress) and has 14 factors, each with a collection of symptoms (physical complaints related to anxiety). Since our study focused only on differentiating normal vs abnormal groups of subjects, the threshold for the scale is acceptable. Each factor is rated on a scale of $0-4$ (extreme), for a total score range of 0-56, with 17 indicating mild severity, 18-24 indicating mild-to-moderate severity, and 25-56 indicating moderate-to-severe severity. However, the detection of ADy in anxiety disorder is difficult with the existing traditional methods (questionnaires). There are very few articles in the literature that directly studied the association between anxiety and its effect on the autonomic nervous system by measuring the variability in heart rate and respiratory rate during a sleep recording [4], [5]. Therefore, the current study aimed to develop computational tools that can be used for the detection of anxiety disorder by screening ADys from electrocardiogram (ECG) and respiratory signals.

Quantitative evaluation of neurophysiological signals like ECG and respiratory requires the computer-based implementation of estimation algorithms [6], [7]. The computer-based estimations involve accurate estimation of statistical properties from the recorded neurophysiological signals. The ECG signal is made up of the $\mathrm{P}$ wave, QRS complex, $\mathrm{T}$ wave, and $\mathrm{U}$ wave. The time interval between subsequent $\mathrm{R}-\mathrm{R}$ peaks is an important measure estimated from the ECG signal. The variation in the R-R interval between two consecutive heartbeats is called Heart Rate Variability (HRV) [8], [9]. The variation within the respiratory rhythm is termed respiratory rate variability (RRV). Changes in heart rate, blood pressure, and pulse tend to change involuntary activities of the respiratory system, assessing these variations could provide more insight into the analysis of autonomic dysfunction. Similar to the HRV analysis, respiratory signals can be quantified based on the variability in measurements of successive breaths. The respiratory rate can be evaluated based on the number of breaths a person takes per minute. The respiratory rate of a healthy adult at rest is between 12 and 18 breaths per minute.

Researchers use time domain, frequency domain, and non-linear indices to measure the variability in ECG and respiratory signals [8]. In the time domain, BPM (beats in a minute), SDNN (The standard deviation of NN intervals), RMSD (Root-mean-square of successive RR interval differences) and SDSD (The standard deviation of successive $\mathrm{R}-\mathrm{R}$ intervals), etc. were computed to study the statistical property of the signal. RR and NN denote the interval between two heartbeats ( $\mathrm{R}$ spikes in the QRS complex / ECG). On other hand, the frequency-domain measurement estimates the distribution of absolute or relative power into four frequency bands: low-frequency (LF), and highfrequency (HF) bands. The variability in HF and LF can be calculated from the estimated power spectrum and area under the curve. The noise or artefacts in the signal can 
interfere with the spectral analysis of these parameters. In those scenarios, careful inspection and exclusion of those segments or advanced signal processing techniques can be used to overcome such interferences.

Two important questions we examined in the study were, 1) Do ECG and respiratory signals recorded during a sleep study can identify subjects with an anxiety disorder? 2) How to develop computational solutions for screening of ADy and prevent serious events like coronary heart disease in subjects with anxiety disorder? We know that Machine learning (ML) is a powerful computational technique that can assist in the diagnosis of several diseases [10]-[15]. To attempt and address these questions, we developed ML models to predict ADy in anxiety patients using ECG and respiratory signals recorded during a sleep study in the clinic. 23 features were developed from neurophysiological signals to the classification of subjects. The accuracy of various supervised ML algorithms (SVM, KNN, Logistic Regression, and ensemble-based approaches) in predicting autonomic dysfunction in anxiety were compared in the study.

\section{Methods}

\subsection{Data collection}

In our study, 52 subjects with suspected anxiety disorder were included, evaluated from Jan 2019 to Dec 2019. The subjects were evaluated based on Hamilton Anxiety Rating (HAM-A) and were grouped into two. Group 1 consists of normal Individuals (HAM-A score < 18) and group 2 consists of subjects with possible autonomic function disorders (HAM-A score > 18). All subjects underwent sleep study as part of clinical evaluation. All signals were recorded at $256 \mathrm{~Hz}$ using the Natus Brain Monitor model 2017 and the ECG signal was obtained by calculating the voltage difference between ECG-L and ECG-R. To obtain the respiratory signal, the respiratory flow channel measurement was used. The institutional review board has approved the study design.

\subsection{Data preprocessing}

ECG and respiratory signals are prone to be affected by various artefacts including baseline wander, power line noise, and electromyographic noise. Baseline wander is a low-frequency noise in the frequency band from 0.5 to $0.6 \mathrm{~Hz}$ that occurred due to the movement of electrodes during the patient movements or breathing [12]. Power line interference was caused by electrical appliances and may introduce $50 \mathrm{~Hz}$ frequency noises in the signal. All these noises can lead to a wrong interpretation of physiological signals and functions. In our study, digital filters were used to remove artefacts from the recordings, a notch filter at $50 \mathrm{~Hz}$ was used to remove the power line interferences and a kaiser window coefficients and finite impulse response filter were used to eliminate the baseline drift [13]. 


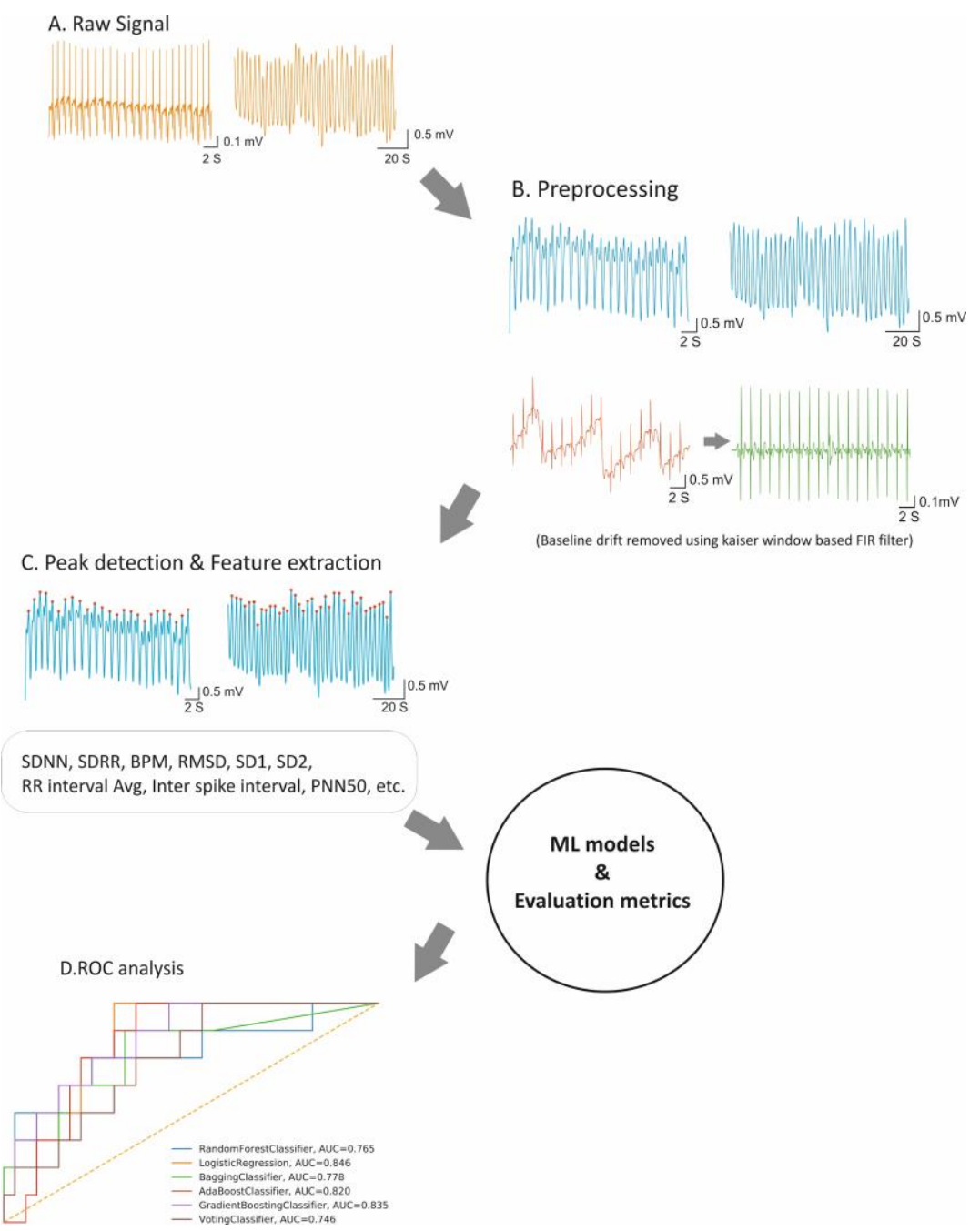

Fig. 1. Schematic of the study. (A) Raw ECG and respiratory signal, (B) Preprocessed ECG and respiratory signal. (C) \& (D) Estimation of statistical measures (Table 1, 2 and 3), (D) ML model training and validations using AUC ROC curve.

\subsection{Building ML models to predict the ADy in anxiety disorder}

In this study, supervised ML models were used to predict the ADy in anxiety disorder. Preprocessed ECG and respiratory signals were used to estimate the ML features. Linear, nonlinear metrics and frequency-domain parameters were estimated to train the classifier. Figure 2 shows the estimated frequency-domain parameters from ECG signal using the welch method for computing power spectral density [14]. Table 1, 2 and 3 describes the features considered for the study along with the equations. The true label was assigned to each dataset based on the questionnaire score. A HAM-A score below 
18 was labelled as group 1 (subjects without anxiety) and HAM-A scores above 18 were labelled as group 2 (subjects with anxiety).

The availability of large datasets for mathematical evaluation of our disease group was limited. In order to overcome such limitations, we segmented the ECG and respiratory signal into 30 minutes (116 datasets), 10 minutes (139 datasets), and 5 minutes (214 datasets) periods. For each data set, 18 features were estimated for the ECG signal [8], and 5 features were estimated for the respiratory signal. Principal Component Analysis (PCA) transforms the feature dataset into smaller uncorrelated new sets of features called the Principal Component. We performed PCA on the dataset to remove fewer contributing features from multi-dimensional features. The segments contaminated with artifacts were not included in the analysis. Traditional statistical test such as independent sample t-test was applied to compare the mean of features generated from two groups of data sets (see Table 1 and 2).

Python programming language and libraries including sklearn, seaborn, and matplotlib were used to build ML models to classify the subjects with and without anxiety. The python toolbox for Heart Rate Variability, pyHRV was uses to compute the frequency domain measures [15]. A few classifier models were tested on our dataset to choose the best model. ML classifiers and descriptions were given in Table 3. Crossvalidation and Receiver Operating Characteristic curve (ROC) analysis was used to evaluate the prediction accuracy of ML models. The dataset was randomly divided into two sets, $80 \%$ for training and $20 \%$ for tests. The highest prediction accuracy obtained from this set of analysis was called the cross-validation accuracy of the ML model. By calculating the false positive rate and true positive rate for different thresholds, ROC was used to select the classifier's performance.

\section{$3 \quad$ Results}

From 52 subjects, 214 artefact free ECG segments were extracted, 132 were grouped into G1 (normal, score <18) and 82 of them to G2 (abnormal, score > 18). ML methods including SVM, K Nearest Neighbor, Decision tree, and Logistic regression were used to build the anxiety prediction models (see Table 3 ).

To test the statistics between the two groups, an independent sample t-test was performed. Out of 23 features, 11 showed a statistically significant difference in the mean with a p-value $<0.05$. The PCA on the dataset showed $95 \%$ information retention when we include 8 Principal Components (PCs), features were listed in Table 1 and 2. In further analysis, we used 8 PCs for training all models. Segmenting datasets into smaller intervals (5,10 and 30 minutes) yielded an increased number of the dataset for training and testing. ECG and respiratory signal features were used to train ML models for all segmented dataset types. 


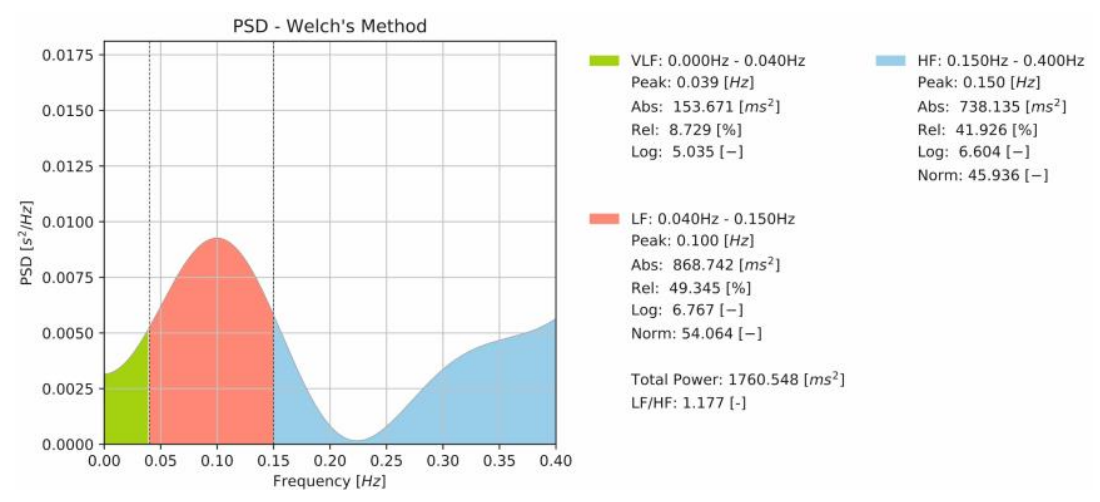

Fig. 2. Estimated frequency domain parameters from ECG signal using power spectral density (PSD). Graph was created using [15].

A.

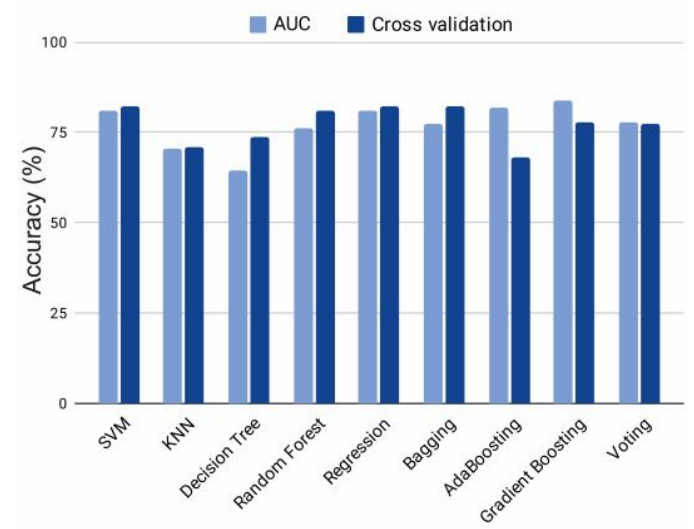

B.

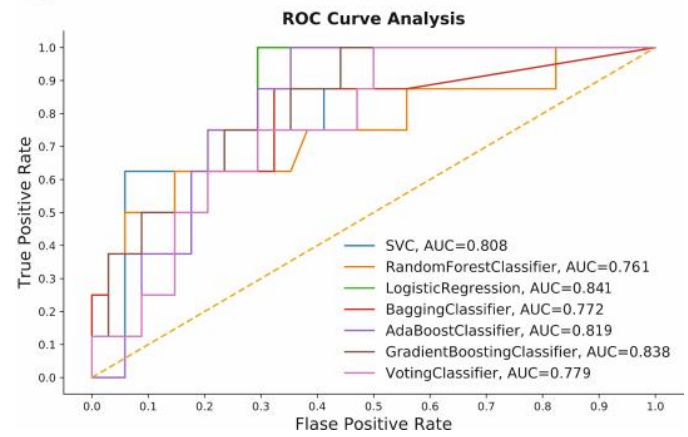

Fig. 3. Validating ML models using Receiver Operating Characteristic curve analysis. (A) Bar plot showing cross validation and AUC when models were trained with 10 minutes segmented dataset, (B) Receiver Operating Characteristic curve analysis. 
In 10 and 30 minutes segmented dataset, SVM, random forest and gradient boosting ensembles gave the best cross-validation accuracy of $82.2 \%, 81.64 \%$ and $79.06 \%$ and AUC of $0.81,0.84$ and $0.86 \%$ for ECG signal (see Figure 3 and Table 4 ). We observed less prediction accuracy when the ML models were trained with features extracted from 5 minutes segmented datasets compared to models trained with 10 and 30 minutes segmented datasets (see Table 4). We also observed that the classification accuracy decreased when models were trained with features combined with ECG and respiratory signals (Table 5).

\section{Discussion}

The main objective of this work was to study the association between anxiety and its effect on the autonomic nervous system by measuring the variability in heart rate and respiratory rate in subjects undergoing a sleep study. In our study, we used a HAM-A score to assign class labels (subjects with and without anxiety disorders) to the input datasets. The features estimated from quantitative neurophysiological signals were used to train the ML models to classify subjects with and without anxiety disorders.

ML models including SVM, KNN, Logistic Regression and ensemble-based approaches were used to classify the subjects with and without anxiety and to assess autonomic dysfunction in these patients. As shown in other studies, in absence of a large number of datasets, the available datasets may be segmented into smaller time intervals and used to test the statistics or train the ML models [16]-[18]. To build generalized ML models, we segmented the signals into 5, 10, and 30-minutes intervals that increase the number of datasets for training and testing. We observed that the random forest, gradient boost ensembles and SVM based classifiers gave the best accuracy for the model trained with features generated from 10 and 30 minutes segmented signals. As reported in previous studies, the SVM and random forest classifier were found to be more apt for classification problems based on physiological signals [19]-[22].

A recent study by Kupats et al, reported that there was no relationship between the symptoms of generalized anxiety and cardiovascular ADy [4]. However, in our results, we observed that 11 out of 23 features estimated from ECG and respiratory signal have shown a significant statistical difference in both anxious and non-anxious groups. These findings indicated that there is a significant autonomic disturbance in the anxious group.

A recent study by Ihmig et al, showed ML models can be used to detect anxiety in subjects with spider fear using ECG and respiratory signals [23]. In the current study, we tested how well the ML models can predict the ADys in anxiety disorder from ECG and respiratory signals recorded during a sleep study. Studies by Chalmers et al and Dekker et al reported that short-term HRV of lengths varying from 2 minutes to 1 hour can be used as datasets of detecting autonomic dysfunction [16]-[18]. In concordance with the previous findings, our results also indicated that segmenting the input signal dataset into 10 minutes and above gave better classification accuracy for anxiety prediction. Our analysis also recommended that analyzing/segmenting neurophysiological signals less than 10 minutes may result in less classification accuracy for ADy diagnosis in anxiety patients.

This study was limited by a smaller number of patient data used for training the models. One way to overcome such a scenario was segmenting the signal into smaller 
periods that increase the number of datasets for training and testing. Another limitation was the acquisition of both respiratory and ECG signals that can easily be distorted by movement and volitional inputs. Advanced sensor-based recording devices need to be developed for the artefact-free acquisition of these signals.

\section{Conclusion}

In this study, with limited datasets, we demonstrated that ML models with features estimated from ECG and respiratory signals can identify ADys in patients with anxiety disorder. Out of 23 features estimated, 11 were found to be statistically significant for the classification of anxious and non-anxious groups. ML models including SVM and random forest classification gave the best classification accuracy. The study also suggests that the classification accuracy was increased when the models were trained with features extracted from only ECG signals. Our results also recommended that segmenting the ECG and respiratory signal by not less than 10 minutes segments for better accuracy in autonomic dysfunction prediction in anxiety disorder. Our recommendations were concordant with reported ranges of ECG segmentation in the literature, except the lower range.

Findings from the current study need to be tested on a large set of patient data to generalize the recommendations. The future direction of the study will be on training ADy dataset on deep neural networks with a larger dataset to produce more generalized models and development of wearable devices. A detailed prospective study also needs to be planned before using the tool in neurology clinics.

Table 1. Features estimated for ECG signal

\begin{tabular}{|c|c|c|c|c|}
\hline Parameter & Equation & $\begin{array}{l}\text { Estimated } \\
\text { value }\end{array}$ & $\begin{array}{c}\text { Re- } \\
\text { ported } \\
\text { Range }\end{array}$ & $\begin{array}{c}\text { t-Test } \\
\text { (p-value) }\end{array}$ \\
\hline $\begin{array}{l}\text { SDNN (ms) } \\
\text { The standard deviation of N-N inter- } \\
\text { vals }\end{array}$ & $\begin{array}{l}\quad=\sqrt{\frac{1}{N} \sum_{i=1}^{N}\left(x_{i}-\mu\right)^{2}} \\
\begin{array}{l}N \text { denotes total time; } \mathrm{X} \text { de- } \\
\text { notes features and } \mu \text { denotes } \\
\text { mean. }\end{array}\end{array}$ & 55 & $32-93$ & $\begin{array}{l}\text { Insignificant } \\
0.3395\end{array}$ \\
\hline $\begin{array}{l}\text { BPM } \\
\text { The average number of beats in a mi- } \\
\text { nute }\end{array}$ & $\begin{array}{l}=\frac{\sum R-\text { peaks }}{\text { duration in minutes }} \\
\text { R-peaks denotes total number } \\
\text { of successive } \mathrm{R} \text {-peaks values }\end{array}$ & 64 & $60-100$ & $\begin{array}{l}\text { Significant } \\
0.0001\end{array}$ \\
\hline $\begin{array}{l}\text { RR interval Avg } \\
\text { Difference between peak-to-peak in- } \\
\text { terval by the total number of peaks }\end{array}$ & $=\frac{\sum R-\text { peaks }}{\text { sampling rate }}$ & 936 & $\begin{array}{l}785- \\
1,160\end{array}$ & $\begin{array}{l}\text { Insignificant } \\
0.4136\end{array}$ \\
\hline $\begin{array}{l}\text { Inter spike interval } \\
\text { Difference between spike-to-spike in- } \\
\text { terval }\end{array}$ & $=\frac{\text { duration }(\mathrm{sec})}{60}$ & 30 & $\begin{array}{c}0.6-1.2 \\
\text { sec }\end{array}$ & $\begin{array}{l}\text { Significant } \\
0.0002\end{array}$ \\
\hline
\end{tabular}


Paper-Predicting Autonomic Dysfunction in Anxiety Disorder from ECG and Respiratory Signals...

\begin{tabular}{|l|c|c|c|l|}
\hline $\begin{array}{l}\text { RMSD (ms) } \\
\text { Root-mean-square of successive R-R } \\
\text { interval differences }\end{array}$ & $=\frac{\sum_{i=1}^{n} R-p e a k_{i+1}-R-p e a h}{n}$ & 44 & $19-75$ & $\begin{array}{l}\text { Significant } \\
0.0008\end{array}$ \\
\hline $\begin{array}{l}\text { SDSD }(m s) \\
\text { The standard deviation of successive } \\
\text { R-R intervals }\end{array}$ & $=\sqrt{\frac{1}{N} \sum_{i=1}^{N}\left(\text { Spike }_{i}-\text { Spike }_{i+}\right.}$ & 21 & - & $\begin{array}{l}\text { Significant } \\
0.0317\end{array}$ \\
\hline Inter Peak Time Interval Max (ms) & Max (Spike $e_{i}-$ Spike $\left._{i+1}\right)$ & 1.19 & 1.2 & $\begin{array}{l}\text { Insignificant } \\
0.05114\end{array}$ \\
\hline Inter Peak Time Interval Min (ms) & Min(Spik $e_{i}-$ Spike $\left._{i+1}\right)$ & 0.6 & 0.6 & $\begin{array}{l}\text { Insignificant } \\
0.3234\end{array}$ \\
\hline
\end{tabular}

Table 2. Estimated frequency domain and non-linear measures of ECG and respiratory signal

\begin{tabular}{|c|c|c|c|c|}
\hline Metrics & Description & $\begin{array}{c}\text { Esti- } \\
\text { mated } \\
\text { value }\end{array}$ & $\begin{array}{l}\text { Reported } \\
\text { range }\end{array}$ & $\begin{array}{c}\text { t-Test } \\
(p-v a l u e)\end{array}$ \\
\hline VLF power(ms2) & $\begin{array}{l}\text { The absolute power of the very-low-frequency } \\
\text { band }\end{array}$ & 34 & - & $\begin{array}{l}\text { Insignificant } \\
0.3659\end{array}$ \\
\hline VLF Peak (Hz) & $\begin{array}{l}\text { The peak frequency of the very low-frequency } \\
\text { band }\end{array}$ & 0.04 & 0.04 & $\begin{array}{l}\text { Significant } \\
0.0472\end{array}$ \\
\hline LF peak $(\mathrm{Hz})$ & The peak frequency of the low-frequency band & 0.1 & $\begin{array}{c}(0.04-0.15 \\
\mathrm{Hz})\end{array}$ & $\begin{array}{l}\text { Insignificant } \\
0.1869\end{array}$ \\
\hline LF power (ms2) & The absolute power of the low-frequency band & 284 & $193-1,009$ & $\begin{array}{l}\text { Significant } \\
0.0017\end{array}$ \\
\hline HF peak $(\mathrm{Hz})$ & The peak frequency of the high-frequency band & 0.15 & $\begin{array}{c}(0.15-0.4 \\
\mathrm{Hz})\end{array}$ & $\begin{array}{l}\text { Insignificant } \\
0.1017\end{array}$ \\
\hline HF power (ms2) & The absolute power of the high-frequency band & 101 & $83-3,630$ & $\begin{array}{l}\text { Significant } \\
0.0036 \\
\end{array}$ \\
\hline $\mathrm{LF} / \mathrm{HF}(\mathrm{ms})$ & The ratio of LF-to-HF power & 1.17 & $1.1-11.6$ & $\begin{array}{l}\text { Insignificant } \\
0.7256\end{array}$ \\
\hline Total power (ms) & Sum of LF, HF, VLF power & 1420 & - & $\begin{array}{l}\text { Significant } \\
0.0159\end{array}$ \\
\hline SD1 & $\begin{array}{l}\text { Standard deviation perpendicular to the line of } \\
\text { identity }\end{array}$ & 35 & - & $\begin{array}{l}\text { Significant } \\
0.0001\end{array}$ \\
\hline SD2 & Standard deviation parallel the line of identity & 68 & - & $\begin{array}{l}\text { Insignificant } \\
0.2407\end{array}$ \\
\hline Parameter (ms) & Description & $\begin{array}{c}\text { Esti- } \\
\text { mated } \\
\text { value }\end{array}$ & $\begin{array}{l}\text { Reported } \\
\text { range }\end{array}$ & $\begin{array}{c}\text { t-Test } \\
\text { (p-value) }\end{array}$ \\
\hline IBI-avg & The time interval between breaths & 2.74 & $\sim 0.4-\sim 3$ & $\begin{array}{l}\text { Significant } \\
0.0299 \\
\end{array}$ \\
\hline IBI-max & The standard deviation of RR intervals & 6.7 & 25 & $\begin{array}{l}\text { Insignificant } \\
0.1206\end{array}$ \\
\hline IBI-min & The minimum of inter breath peak time interval & 2 & 2 & $\begin{array}{l}\text { Insignificant } \\
0.9481\end{array}$ \\
\hline BPT-avg & Breath peak interval average & 9.99 & - & $\begin{array}{l}\text { Insignificant } \\
0.2972 \\
\end{array}$ \\
\hline BPM & Mean of number of breaths in a minute & 16 & $12-20$ & $\begin{array}{l}\text { Significant } \\
0.0020\end{array}$ \\
\hline
\end{tabular}


Paper-Predicting Autonomic Dysfunction in Anxiety Disorder from ECG and Respiratory Signals...

Table 3. List of machine learning models used in the study

\begin{tabular}{|l|l|}
\hline $\begin{array}{c}\text { ML algo- } \\
\text { rithms }\end{array}$ & \multicolumn{1}{c|}{ Description } \\
\hline SVM & $\begin{array}{l}\text { Support Vector Machine is a powerful ML algorithm capable of performing linear and } \\
\text { non-linear regression, and classification on smaller and larger datasets. SVM creates a hy- } \\
\text { perplane to maximize the decision boundary (generalization). The data points that form } \\
\text { the boundary are called support vectors. }\end{array}$ \\
\hline KNN & $\begin{array}{l}\text { K-nearest neighbors (KNN), a lazy learner, supervised machine learning method. The } \\
\text { KNN uses the K value to create a distance metric (using Euclidean distance) and classifies } \\
\text { the K-nearest neighbors of the data. The new data points are categorized based on the ma- } \\
\text { jority number of neighbors. }\end{array}$ \\
\hline $\begin{array}{l}\text { Random } \\
\text { Forest }\end{array}$ & $\begin{array}{l}\text { The random forest algorithm is an ensemble learning method. It uses a combination of de- } \\
\text { cision trees and a voting mechanism to select labels. Due to extra randomness, the random } \\
\text { forest tends to have a better generalization performance enabling in the reduction of model } \\
\text { variance. }\end{array}$ \\
\hline $\begin{array}{l}\text { Logistic } \\
\text { Regres- } \\
\text { sion }\end{array}$ & $\begin{array}{l}\text { Logistic Regression is a supervised learning algorithm that uses categorical data to predict } \\
\text { probability. The target of the algorithm is to figure out a boolean expression that predicts a } \\
\text { binary outcome for the classification. }\end{array}$ \\
\hline Bagging & $\begin{array}{l}\text { Bagging algorithms (or bootstrap aggregation) is a technique where multiple subsets of } \\
\text { data are created to train the model. The output of this model is calculated as averaged } \\
\text { across all the sub models. It works best with algorithms that have low bias and high vari- } \\
\text { ances like SVM and decision trees. Here we have used bootstrap aggregation with SVM } \\
\text { for classification. }\end{array}$ \\
\hline Boosting & $\begin{array}{l}\text { Boosting is an ensemble method that utilizes the combination of several weak learners to } \\
\text { form a stronger learner by creating a sequence of models. }\end{array}$ \\
\hline Voting & $\begin{array}{l}\text { Voting is another ensemble-based approach which uses multiple ML classifiers and uses } \\
\text { the maximum vote to predict the class label. }\end{array}$ \\
\hline
\end{tabular}


Table 4. Results of ML models trained with features extracted from full duration, 5, 10 and 30 minutes ECG segments.

\begin{tabular}{|c|c|c|c|c|c|c|}
\hline \multirow[b]{2}{*}{ Classifiers } & \multicolumn{2}{|c|}{$\begin{array}{l}\text { ML models trained on } 5 \\
\text { minutes ECG segments }\end{array}$} & \multicolumn{2}{|c|}{$\begin{array}{c}\text { ML models trained on } \\
10 \text { minutes ECG seg- } \\
\text { ments }\end{array}$} & \multicolumn{2}{|c|}{$\begin{array}{l}\text { ML models trained on } 30 \\
\text { minutes ECG segments }\end{array}$} \\
\hline & $\begin{array}{c}\text { Cross } \\
\text { validation }\end{array}$ & $\boldsymbol{A U C}$ & Cross validation & $A U C$ & Cross validation & $A U C$ \\
\hline SVM & 76.31 & 0.636 & 82.2 & 0.809 & 78.37 & 0.658 \\
\hline $\mathrm{KNN}$ & 80.03 & 0.7423 & 71.13 & 0.706 & 73.88 & 0.613 \\
\hline Decision Tree & 71.72 & 0.582 & 73.75 & 0.643 & 71.46 & 0.654 \\
\hline Random Forest & 72.14 & 0.537 & 81.02 & 0.761 & 81.64 & 0.852 \\
\hline $\begin{array}{l}\text { Logistic } \\
\text { Regression }\end{array}$ & 82.83 & 0.771 & 75.91 & 0.842 & 79.13 & 0.708 \\
\hline Bagging & 75.85 & 0.686 & 82.2 & 0.772 & 81.78 & 0.702 \\
\hline AdaBoost & 67.85 & 0.680 & 68.05 & 0.820 & 74.6 & 0.792 \\
\hline $\begin{array}{l}\text { Gradient } \\
\text { Boosting }\end{array}$ & 71.66 & 0.628 & 78 & 0.838 & 79.06 & 0.858 \\
\hline Voting & 77.24 & 0.692 & 77.33 & 0.779 & 78.36 & 0.883 \\
\hline
\end{tabular}

Table 5. Results of ML models trained with features extracted from 10 minutes segmented ECG and Respiratory signals combined.

\begin{tabular}{|l|c|c|c|c|}
\hline & \multicolumn{2}{|c|}{ ML models trained on Respiratory signal } & \multicolumn{2}{c|}{$\begin{array}{c}\text { ML models trained on ECG and } \\
\text { Respiratory signal }\end{array}$} \\
\hline \multicolumn{1}{|c|}{ Classifiers } & Cross validation & AUC & Cross validation & AUC \\
\hline SVM & 75.04 & 0.371 & 73.82 & 0.724 \\
\hline KNN & 78.11 & 0.496 & 76.35 & 0.737 \\
\hline Decision Tree & 67.77 & 0.474 & 71.71 & 0.706 \\
\hline Random Forest & 76.73 & 0.578 & 77.45 & 0.667 \\
\hline Logistic Regression & 82.45 & 0.636 & 76.11 & 0.805 \\
\hline Bagging & 76.52 & 0.522 & 75.45 & 0.651 \\
\hline AdaBoost & 67.41 & 0.588 & 70.38 & 0.625 \\
\hline Gradient Boosting & 70.23 & 0.518 & 74.06 & 0.761 \\
\hline Voting & 80.31 & 0.511 & 76.78 & 0.794 \\
\hline
\end{tabular}

\section{Acknowledgement}

We thank sleep lab technologists, Ms. Sonu, Ms. Minnu, Ms. Radika and Ms. Renuka for their help in data collection. This study was supported by the seed grant for research from Amrita Institute of Medical Sciences, India. 


\section{$7 \quad$ References}

[1] A. Steptoe, "Psychological Methods: An Overview of Clinical Applications," in Breakdown in Human Adaptation to 'Stress',' Springer Netherlands, 1984, pp. 208-218. https://doi. org/10.1007/978-94-011-8064-1_14

[2] David H. Barlow, Anxiety and Its Disorders: The Nature and Treatment of Anxiety and Panic, 2nd ed. Guilford Press, 2004.

[3] A. Gjerris et al., "The Hamilton Anxiety Scale. Evaluation of homogeneity and inter-observer reliability in patients with depressive disorders," Journal of Affective Disorders, vol. 5, no. 2, pp. 163-170, May 1983, https://doi.org/10.1016/0165-0327(83)90009-5

[4] E. Kupats, I. Noviks, J. Vrublevska, V. Kenina, U. Kojalo, and I. Logina, "No relationship between generalised anxiety symptoms and cardiovascular autonomic dysfunction," Neurology Psychiatry and Brain Research, vol. 30, pp. 86-90, Dec. 2018, https://doi. org/10.1016/j.npbr.2018.07.001

[5] G. A. Alvares, D. S. Quintana, I. B. Hickie, and A. J. Guastella, “Autonomic nervous system dysfunction in psychiatric disorders and the impact of psychotropic medications: A systematic review and meta-analysis," Journal of Psychiatry and Neuroscience, vol. 41, no. 2, pp. 89-104, Mar. 2016, https://doi.org/10.1503/ipn.140217

[6] F. Lotte et al., "A review of classification algorithms for EEG-based brain-computer interfaces: A 10 year update," Journal of Neural Engineering, vol. 15, no. 3. Institute of Physics Publishing, Apr. 2018. https://doi.org/10.1088/1741-2552/aab2f2

[7] G. Giannakakis, D. Grigoriadis, K. Giannakaki, O. Simantiraki, A. Roniotis, and M. Tsiknakis, "Review on psychological stress detection using biosignals," IEEE Transactions on Affective Computing, 2019, https://doi.org/10.1109/taffc.2019.2927337.

[8] F. Shaffer and J. P. Ginsberg, "An Overview of Heart Rate Variability Metrics and Norms," Frontiers in Public Health, vol. 5, p. 258, Sep. 2017, https://doi.org/10.3389/fpubh. 2017.00258

[9] M. Hammad, A. Maher, K. Wang, F. Jiang, and M. Amrani, "Detection of abnormal heart conditions based on characteristics of ECG signals," Measurement: Journal of the International Measurement Confederation, vol. 125, pp. 634-644, Sep. 2018, https://doi.org/10. 1016/j.measurement.2018.05.033

[10] U. Raghavendra, U. R. Acharya, and H. Adeli, "Artificial Intelligence Techniques for Automated Diagnosis of Neurological Disorders," European Neurology, vol. 82, no. 1-3. S. Karger AG, pp. 41-64, Feb. 2020. https://doi.org/10.1159/000504292

[11] J. Wiens and E. S. Shenoy, "Machine Learning for Healthcare: On the Verge of a Major Shift in Healthcare Epidemiology," Clinical Infectious Diseases, vol. 66, no. 1, pp. 149-153, Jan. 2018, https://doi.org/10.1093/cid/cix731

[12] R. Kher, "Signal Processing Techniques for Removing Noise from ECG Signals," J. Biomed. Eng. Res., vol. 3, pp. 1-9, 2019.

[13] M. S. Chavan, R. A. Agarwala, and M. D. Uplane, "Use of Kaiser window for ECG processing," Proceedings of the 5th WSEAS International Conference on Signal Processing, Robotics and Automation, vol. 2006, no. January 2006, pp. 285-289, 2006.

[14] P. D. Welch, "The Use of Fast Fourier Transform for the Estimation of Power Spectra: A Method Based on Time Averaging Over Short, Modified Periodograms," IEEE Transactions on Audio and Electroacoustics, vol. 15, no. 2, pp. 70-73, 1967, https://doi.org/10.1109/ tau.1967.1161901

[15] P. M. C. Gomes, "Development of an Open-Source Python Toolbox for Heart Rate Variability (pyHRV)," Proc. Int'l Conf. on Electrical, Electronic and Computing Engineering (IcETRAN), pp. 822-828, 2018.

[16] J. A. Chalmers, D. S. Quintana, M. J. A. Abbott, and A. H. Kemp, "Anxiety disorders are associated with reduced heart rate variability: A meta-analysis," Frontiers in Psychiatry, vol. 5, no. JUL, pp. 1-11, 2014, https://doi.org/10.3389/fpsyt.2014.00080 
[17] H. Tsuji et al., "Impact of reduced heart rate variability on risk for cardiac events: The Framingham Heart Study," Circulation, vol. 94, no. 11, pp. 2850-2855, 1996, https://doi.org/ 10.1161/01.cir.94.11.2850

[18] J. M. Dekker et al., "Low heart rate variability in a 2-minute rhythm strip predicts risk of coronary heart disease and mortality from several causes: The ARIC study," Circulation, vol. 102, no. 11, pp. 1239-1244, Sep. 2000, https://doi.org/10.1161/01.cir.102.11.1239

[19] H. Parasuram, S. KrishnaA, V. GopalK, and P. K. Krishnan Namboori, "Machine Learning Approaches to Determine the 'Drug-Likeness' of the Proteomic Targets,", Proc. of Int. Conf. on Control, Communication and Power Engineering 2010.

[20] A. Kampouraki, G. Manis, and C. Nikou, "Heartbeat time series classification with support vector machines," in IEEE Transactions on Information Technology in Biomedicine, 2009, vol. 13, no. 4, pp. 512-518. https://doi.org/10.1109/titb.2008.2003323

[21] R. Ani, G. Sasi, U. R. Sankar, and O. S. Deepa, "Decision support system for diagnosis and prediction of chronic renal failure using random subspace classification," in 2016 International Conference on Advances in Computing, Communications and Informatics, ICACCI 2016, 2016, pp. 1287-1292. https://doi.org/10.1109/icacci.2016.7732224

[22] S. Vattamthanam, G. B. Mrudula, and C. S. Kumar, "Sleep Apnea Classification Using Deep Neural Network," in 2020 IEEE International Conference on Distributed Computing, VLSI, Electrical Circuits and Robotics, DISCOVER 2020 - Proceedings, 2020, pp. 133-136. https://doi.org/10.1109/discover50404.2020.9278045

[23] F. R. Ihmig, A. G. H., F. Neurohr-Parakenings, S. K. Schäfer, J. Lass-Hennemann, and T. Michael, "On-line anxiety level detection from biosignals: Machine learning based on a randomized controlled trial with spider-fearful individuals," PLOS ONE, vol. 15, no. 6, p. e0231517, Jun. 2020, https://doi.org/10.1371/journal.pone.0231517

\section{Authors}

Abhilash Saj George is a Master of Computer Application student at the Department of Computer Science and Applications, Amrita Vishwa Vidyapeetham, Amritapuri, India.

Arjun Vijayanatha Kurup is a Master of Computer Application student at the Department of Computer Science and Applications, Amrita Vishwa Vidyapeetham, Amritapuri, India.

Parthasarathy Balachandran is a DM resident at the Department of Neurology, Amrita Institute of Medical Sciences, Kochi, Kerala, India.

Manjusha Nair is an Assistant Professor at the Department of Computer Science and Applications, Amrita Vishwa Vidyapeetham, Amritapuri, India.

Siby Gopinath is a Neurologist and Professor at the Department of Neurology, Amrita Institute of Medical Sciences, Kochi, Kerala, India.

Anand Kumar is a Neurologist, Professor and HOD at the Department of Neurology, Amrita Institute of Medical Sciences, Kochi, Kerala, India.

Harilal Parasuram is a Computational Neurologist and Assistant Professor at the Department of Neurology, Amrita Institute of Medical Sciences, Kochi, Kerala, India.

Article submitted 2021-03-12. Resubmitted 2021-05-20. Final acceptance 2021-05-20. Final version published as submitted by the authors. 